ELECTRONIC RESEARCH ANNOUNCEMENTS OF THE AMERICAN MATHEMATICAL SOCIETY

Volume 5, Pages 128-135 (October 29, 1999)

S $1079-6762(99) 00071-2$

\title{
EXPONENTIAL SUMS WITH MULTIPLICATIVE COEFFICIENTS
}

\author{
GENNADY BACHMAN
}

(Communicated by Hugh Montgomery)

AbStract. We provide estimates for the exponential sum

$$
F(x, \alpha)=\sum_{n \leq x} f(n) e^{2 \pi i \alpha n},
$$

where $x$ and $\alpha$ are real numbers and $f$ is a multiplicative function satisfying $|f| \leq 1$. Our main focus is the class of functions $f$ which are supported on the positive proportion of primes up to $x$ in the sense that $\sum_{p \leq x}|f(p)| / p \gg$ $\log \log x$. For such $f$ we obtain rather sharp estimates for $F(x, \alpha)$ by extending earlier results of H. L. Montgomery and R. C. Vaughan. Our results provide a partial answer to a question posed by G. Tenenbaum concerning such estimates.

Estimating exponential sums

$$
F(x, \alpha)=\sum_{n \leq x} f(n) e(\alpha n) \quad(x \geq 3, \quad \alpha \in \mathbb{R}),
$$

where $f$ is a multiplicative function and $e(t)$ stands for $e^{2 \pi i t}$, is an interesting problem which has received considerable attention in analytic number theory. Roughly, the research on this topic is split into two categories. On the one hand, there are estimates for $F(x, \alpha)$ concerned with the particular choices of the function $f$. On the other hand, there are estimates for $F(x, \alpha)$ valid uniformly for all $f$ belonging to some class of multiplicative functions. The main purpose of this note is to announce some new results of the latter kind. We begin, however, by surveying some known estimates for $F(x, \alpha)$.

The simplest instance of our problem is the case when the function $f$ is identically equal to 1 . In this case $F(x, \alpha)$ is a partial sum of a geometric series and we immediately get the bound

$$
F(x, \alpha) \ll \min \left(x, \frac{1}{|e(\alpha)-1|}\right) \ll \min \left(x, \frac{1}{\|\alpha\|}\right),
$$

where $\|\alpha\|$ denotes the distance from $\alpha$ to the nearest integer. It is also immediate that this upper bound is best possible, and so this case is, indeed, trivial. Any other "natural" choice of the function $f$ leads to non-trivial considerations.

Received by the editors June 22, 1998 and, in revised form, October 11, 1999.

1991 Mathematics Subject Classification. Primary 11L07, 11N37.

The author would like to thank Professors Andrew Granville and Gérald Tenenbaum for helpful discussions about various topics related to this project. He especially wishes to thank Professor Adolf Hildebrand for suggesting this problem in the first place, and for numerous discussions on this and related topics over the course of this project. 
Perhaps the most thoroughly studied cases are when the function $f$ is closely related to the Möbius function $\mu$ or is the characteristic function of smooth numbers. A classic result of $\mathrm{H}$. Davenport [Da] states that

$$
\sum_{n \leq x} \mu(n) e(\alpha n) \ll_{A} \frac{x}{(\log x)^{A}},
$$

for any real number $A$. More recently this result has been extended as follows. Let $\mathcal{P}$ denote a set of primes and let $u_{\mathcal{P}}$ be the characteristic function of the set of natural numbers composed entirely of prime factors from $\mathcal{P}$, i.e., $u_{\mathcal{P}}$ is the completely multiplicative function whose values on primes is given by $u_{\mathcal{P}}(p)=1$, if $p \in \mathcal{P}$, and 0 , otherwise. H. L. Montgomery and R. C. Vaughan [MV1] have shown that

$$
\max _{\mathcal{P}, \alpha}\left|\sum_{n \leq x}\left(u_{\mathcal{P}} \mu\right)(n) e(\alpha n)\right| \asymp \frac{x}{\sqrt{\log x}}
$$

This was later strengthened by the author [Ba1] to an asymptotic estimate

$$
\max _{\mathcal{P}, \alpha}\left|\sum_{n \leq x}\left(u_{\mathcal{P}} \mu\right)(n) e(\alpha n)\right|=B \frac{x}{\sqrt{\log x}}\left(1+O\left(\frac{\log \log x}{\sqrt{\log x}}\right)\right),
$$

for some constant $B>0$.

The study of exponential sums over smooth numbers

$$
E(x, y ; \alpha)=\sum_{n \leq x} u_{\mathcal{P}}(n) e(\alpha n) \quad\left(\mathcal{P}=\mathcal{P}_{y}=\{p \leq y\}\right)
$$

was initiated by Vaughan [Va1] (as a special case of a more general exponential sum). Recently E. Fouvry and G. Tenenbaum [FT] obtained sharp estimates for $E(x, y ; \alpha)$. In particular, they established the following bound. Let real numbers $\delta>0$ and $C>0$ be fixed, and let $x \geq 3$ and $y$ satisfy $x^{\delta \log _{3} x / \log _{2} x} \leq y \leq x$, where $\log _{k} x, k=2,3$, denotes the $k$-th iterate of the logarithm function. Then there exists a constant $D=D(\delta, C)$ such that if $Q=x(\log y)^{-D}$ and if $a$ and $q$ are coprime integers satisfying $2 \leq q \leq Q$ and $|\alpha-a / q| \leq 1 /(q Q)$, then we have

$$
E(x, y ; \alpha) \ll_{\delta, C} \Psi(x, y)\left\{\frac{2^{\omega(q)} \log q}{\varphi(q)} \frac{\log (1+\log x / \log y)}{\log y}+\frac{1}{(\log y)^{A}}\right\},
$$

where, as usual, $\Psi(x, y)=\sum_{n \leq x} u_{\mathcal{P}}(n)$. We remark that an estimate for $E(x, y ; \alpha)$ in terms of elementary functions of $x$ and $y$ is readily deduced from this by an appeal to known estimates for $\Psi(x, y)$ (the reader is referred to [HT] for a wonderful survey of this topic).

We now turn to the problem of obtaining estimates for $F(x, \alpha)$ valid uniformly for all $f$ belonging to some class of multiplicative functions. Such a problem was first considered by H. Daboussi for the class $\mathcal{F}$ of all complex-valued multiplicative functions $f$ satisfying $|f| \leq 1$. He showed [Da1] (see also [DD1] and [DD2]) that if $|\alpha-s / r| \leq 1 / r^{2}$ and $3 \leq r \leq(x / \log x)^{1 / 2}$, for some coprime integers $s$ and $r$, then

$$
F(x, \alpha) \ll \frac{x}{\sqrt{\log _{2} r}},
$$


uniformly for all $f \in \mathcal{F}$. This implies, in particular, that for every irrational $\alpha$ we have

$$
\lim _{x \rightarrow \infty} \frac{1}{x} F(x, \alpha)=0,
$$

uniformly for all $f \in \mathcal{F}$. It was later observed by Tenenbaum [Te] that this result provides some measure of independence of the additive and multiplicative structures of the set of integers, a topic of great interest in number theory. More precisely, he formulated the following question. Writing

$$
\frac{1}{x} F(x, \alpha)=\left(\frac{1}{x} \sum_{n \leq x} f(n)\right)\left(\frac{1}{x} \sum_{n \leq x} e(\alpha n)\right)+o(1),
$$

we ask what can be said about the error term. In particular, we would like to characterize those functions $f$ such that for every irrational $\alpha$ we have

$$
\frac{1}{x} F(x, \alpha)=o\left(\frac{1}{x}|F(x, 0)|\right) .
$$

(Observe that (2) implies (3) only for those functions $f$ for which $F(x, 0) \asymp x$.) The question of when (3) holds was first raised in a paper of Y. Dupain, R. R. Hall and Tenenbaum [DHT]. It was shown there, among other things, that (3) holds for the special case of the function $f$ given by $n \mapsto y^{\Omega(n)}$, where $\Omega(n)$ denotes the total number of prime factors of $n$ and $0<y<2$ is fixed. It was also mentioned there that Vaughan [Va2] showed that (3) certainly does not hold for the entire class $\mathcal{F}$. More precisely, he provided an example of a non-negative function $f \in \mathcal{F}$ for which (3) fails and which satisfies the condition

$$
F(x, 0)=\sum_{n \leq x} f(n) \asymp \frac{x}{\log x} .
$$

The problem of characterizing functions $f$ for which the asymptotic relation (3), or even the relation

$$
F(x, \alpha)=o\left(\sum_{n \leq x}|f(n)|\right),
$$

holds appears to be a rather difficult one (note that Vaughan's example applies to $(3)^{\prime}$ as well), and the known results are rather weak. It was shown by Daboussi [Da2] that if $0<y<2$ is fixed, and if $f$ is a completely multiplicative function with $|f(p)|=y$ for all primes $p$, then for every irrational $\alpha$ the relation $(3)^{\prime}$ holds. This was improved by L. Goubin [Go] who showed that the same conclusion holds for any multiplicative function $f$ satisfying the three conditions:

(i) $|f(p)| \leq y$ for every prime $p$,

(ii) the series $\sum_{p} \sum_{\nu=2}^{\infty} \frac{\left|f\left(p^{\nu}\right)\right|}{p^{\nu}}\left(\log p^{\nu}\right)^{\max (1-y, 0)}$ converges,

(iii) the series $\sum_{p} \frac{y-|f(p)|}{p}$ converges.

On the other hand, a spectacular advance was achieved by H. L. Montgomery and R. C. Vaughan [MV2] who improved the original estimate of Daboussi (1) as follows. 
Suppose that $|\alpha-s / r| \leq 1 / r^{2}$ and $2 \leq R \leq r \leq x / R$ for some coprime integers $s$ and $r$. Then we have

$$
F(x, \alpha) \ll \frac{x}{\log x}+\frac{x}{\sqrt{R}}(\log R)^{3 / 2},
$$

uniformly for all $f \in \mathcal{F}$. Furthermore, they showed that aside from the logarithmic factor this bound is sharp. For example, they proved that for any $x \geq 3$ and coprime integers $s$ and $r \geq 1$ there is $f \in \mathcal{F}$ for which

$$
F\left(x, \frac{s}{r}\right) \gg \frac{x}{\log x}+\frac{x}{\sqrt{r}} .
$$

Motivated by (4) we can propose a quantitative form of $(3)^{\prime}$ as follows. Characterize those functions $f$ for which the inequality

$$
F(x, \alpha) \ll \frac{x}{\log x}+\frac{1}{\sqrt{R}} \sum_{n \leq x}|f(n)|
$$

holds, where $x, \alpha$ and $R$ satisfy the hypothesis of (4). Let us immediately note that (6) certainly does not hold for all $f \in \mathcal{F}$. This follows readily by fixing a natural number $r$ and considering a completely multiplicative function $f$ whose values on primes are given by $f(p)=1$, if $p \equiv 1(\bmod r)$, and 0 , otherwise. Thus

$$
F\left(x, \frac{1}{r}\right)=e\left(\frac{1}{r}\right) \sum_{n \leq x} f(n)=e\left(\frac{1}{r}\right) \sum_{\substack{n \leq x \\ p \mid n \Rightarrow p \equiv 1(\bmod r)}} 1,
$$

and hence (6) with $R=r$ will hold only if the last summation over $n$ is $\ll x / \log x$. But, for $x$ sufficiently large, one readily deduces using standard methods the relation

$$
\sum_{\substack{n \leq x \\ p \mid n \Rightarrow p \equiv 1(\bmod r)}} 1 \asymp \frac{x}{\varphi(r)}(\log x)^{\frac{1}{\varphi(r)}-1},
$$

where $\varphi$ is Euler's totient function.

Let us now consider another hybrid of $(3)^{\prime}$ and (4). To this end, we first mention the following simple and very useful estimate of Hall [Ha] (see also $[\mathrm{HR}]$ ). Let $\mathcal{G}$ be the class of all non-negative multiplicative functions $g$ satisfying $0 \leq g \leq 1$. We have

$$
\sum_{n \leq x} g(n) \ll \frac{x}{\log x} \exp \left(\sum_{p \leq x} \frac{g(p)}{p}\right),
$$

uniformly for all $g \in \mathcal{G}$. Furthermore, this estimate is sharp in the sense that there are functions $g \in \mathcal{G}$ for which (7) holds with $\ll$ replaced by $\gg$. This suggests reformulating (6) by replacing the summation over $n$ on the right-hand side by its estimate given by (7). Thus, we want to characterize those functions $f$ for which the inequality

$$
F(x, \alpha) \ll \frac{x}{\log x}+\frac{x}{\sqrt{R} \log x} e^{S(x)}
$$

holds, where $x, \alpha$ and $R$ satisfy the hypothesis of (4) and $S(x)$ is defined by

$$
S(x)=\sum_{p \leq x} \frac{|f(p)|}{p} .
$$


Observe that, by Mertens' estimate, (8) is equivalent to the bound

$$
F(x, \alpha) \ll \frac{x}{\log x}+\frac{x}{\sqrt{R}} \exp \left(-\sum_{p \leq x} \frac{1-|f(p)|}{p}\right),
$$

a form in which a potential improvement over (4) is plain.

A step in the direction of (8) was taken in [Ba2] where in place of (8) the author obtained the weaker bound

$$
F(x, \alpha) \ll_{\epsilon} \frac{x}{\log x}+\frac{x}{R^{1 / 2-\epsilon} \log x} e^{S(x)}\left(\log _{2} x\right)^{1 / 2} .
$$

We are now in the position to overcome the main deficiencies in this estimate [Ba3]. Before stating our new results we introduce some additional notation. Given a function $f \in \mathcal{F}$ and a natural number $q$ we set, analogously to (9),

$$
S_{q}(x)=\sum_{\substack{p \leq x \\(p, q)=1}} \frac{|f(p)|}{p} .
$$

Furthermore, for any real numbers $x \geq 3$ and $\lambda, 0<\lambda \leq 1$, we let $\mathcal{F}_{\lambda}(x)$ be the subclass of $\mathcal{F}$ consisting of functions $f$ satisfying

$$
S(x)=\sum_{p \leq x} \frac{|f(p)|}{p} \geq \lambda \log _{2} x .
$$

Theorem 1. Let $x \geq 3, \alpha$ and $\epsilon>0$ be real numbers and set $Q=x /(\log x)^{3}$. Furthermore, let $a$ and $q$ be coprime integers satisfying $q \leq Q$ and $|\alpha-a / q| \leq$ $1 /(q Q)$. Then we have

$$
F(x, \alpha) \ll_{\epsilon} \frac{x}{\log x}+\frac{x}{\sqrt{q}(\log x)^{1-\epsilon}}+\frac{x}{\sqrt{q} \log x} e^{S_{q}(x)}\left(\frac{q}{\varphi(q)}\right)^{3 / 2},
$$

uniformly for all $f \in \mathcal{F}$.

We extend the range of applicability by proving a somewhat weaker bound as follows.

Theorem 2. Let $x \geq 3, \alpha, R \geq 3$ and $\epsilon>0$ be real numbers and suppose that $|\alpha-s / r| \leq 1 / r^{2}$ and $R \leq r \leq x / R$ for some coprime integers $s$ and $r$. Then we have

$$
F(x, \alpha) \ll_{\epsilon} \frac{x}{\log x}+\frac{x}{\sqrt{R}(\log x)^{1-\epsilon}}+\frac{x}{\sqrt{R} \log x} e^{S(x)}(\log R)^{1 / 2}\left(\log _{2} R\right)^{3 / 2},
$$

uniformly for all $f \in \mathcal{F}$.

Observe that these theorems might be weaker than (10) in those cases when $S(x)$ is "small", e.g., $S(x) \asymp \log _{3} x$. This shortcoming is especially true in view of the fact that we can now replace (10) by the stronger estimate

$$
F(x, \alpha) \ll \frac{x}{\log x}+\frac{x}{\sqrt{R} \log x} e^{S(x)}(\log R)^{1 / 2}\left(\log _{2} R\right)\left(\log _{2} x\right)^{1 / 2} .
$$

Thus by combining these results one obtains a bound superior, in general, to each of them individually. On the other hand, for functions $f$ which are supported on the positive proportion of primes up to $x$, i.e., for $f \in \mathcal{F}_{\lambda}(x)$ for some $0<\lambda \leq 1$, we obtain the following corollaries of Theorems 1 and 2 respectively. 
Corollary 1. Let $x \geq 3$ and $\alpha$ be real numbers and set $Q=x /(\log x)^{3}$. Furthermore, let $a$ and $q$ be coprime integers satisfying $q \leq Q$ and $|\alpha-a / q| \leq 1 /(q Q)$. Then we have

$$
F(x, \alpha) \ll_{\lambda} \frac{x}{\log x}+\frac{x}{\sqrt{q} \log x} e^{S_{q}(x)}\left(\frac{q}{\varphi(q)}\right)^{3 / 2},
$$

uniformly for all $f \in \mathcal{F}_{\lambda}(x)$.

Corollary 2. Let $x \geq 3, \alpha$ and $R \geq 3$ be real numbers and suppose that $|\alpha-s / r| \leq$ $1 / r^{2}$ and $R \leq r \leq x / R$ for some coprime integers $s$ and $r$. Then we have

$$
F(x, \alpha) \ll_{\lambda} \frac{x}{\log x}+\frac{x}{\sqrt{R} \log x} e^{S(x)}(\log R)^{1 / 2}\left(\log _{2} R\right)^{3 / 2},
$$

uniformly for all $f \in \mathcal{F}_{\lambda}(x)$.

The equivalence of estimates (8) and $(8)^{\prime}$ shows that Corollary 2 provides a stronger bound than (4) even in the case when $S(x)$ is maximal. In particular, this shows that our estimates are quite sharp, since we already noted that (4) was. Furthermore, given $\lambda, 0<\lambda \leq 1$, the original examples of Montgomery and Vaughan yielding (5) can be easily modified to produce a function $f \in \mathcal{F}$ for which the analogue of (5)

$$
F\left(x, \frac{s}{r}\right) \gg \frac{x}{\log x}+\frac{x}{\sqrt{r} \log x} e^{S(x)}
$$

holds with $S(x) \sim \lambda \log _{2} x$. Thus we summarize these facts somewhat colloquially by saying that our estimates are sharp "throughout" the class of functions supported on the positive proportion of primes up to $x$ in the sense that (4) is sharp only for the subclass $\mathcal{F}_{1}(x)$.

On the other hand, our results do not imply either (6) or $(3)^{\prime}$. Of course, we do get a slightly weaker form of (6) for those functions $f \in \mathcal{F}_{\lambda}(x)$ for which

$$
\sum_{n \leq x}|f(n)| \asymp \frac{x}{\log x} e^{S(x)} .
$$

Thus, for example, one readily sees using standard methods that functions $f$ for which Daboussi and Goubin established $(3)^{\prime}$ (with $0<y \leq 1$ ) do satisfy this condition. Nevertheless, results we have obtained as well as certain heuristic considerations suggest that the condition $S(x) \gg \log _{2} x$ should go a long way towards guaranteeing that (6) holds. We thus formulate the following conjecture.

Conjecture 1. Let $x \geq 3, \alpha$ and $R \geq 3$ be real numbers and suppose that $\mid \alpha-$ $s / r \mid \leq 1 / r^{2}$ and $R \leq r \leq x / R$ for some coprime integers $s$ and $r$. Then we have

$$
F(x, \alpha) \ll_{\lambda} \frac{x}{\log x}+\frac{x}{\sqrt{R}} \sum_{n \leq x}|f(n)|,
$$

uniformly for all $f \in \mathcal{F}_{\lambda}(x)$.

We also wish to mention a related conjecture, a problem we believe to be of substantial interest in its own right. To this end, let us now consider the problem of determining conditions under which a bound of the form

$$
\sum_{\substack{n \leq x \\ n \equiv a(\bmod q)}} g(n) \ll \frac{1}{\varphi(q)} \sum_{\substack{n \leq x \\(n, q)=1}} g(n)
$$


holds, where $(a, q)=1$ and $g$ is a non-negative multiplicative function satisfying $0 \leq g \leq 1$, i.e., $g \in \mathcal{G}$. Such a result would constitute a multiplicative function analogue of the Brun-Titchmarsh theorem and would certainly be of fundamental importance in the theory of multiplicative functions. Our present interest in this problem stems from the fact that our problem of estimating the exponential sum $F(x, \alpha)$ is reducible to obtaining estimates of a kind given by (11). Observe that the need for such a result for the purposes of estimating $F(x, \alpha)$ ties in well with the observations of Tenenbaum we gave earlier, since (11) is certainly a result on the independence of the additive and multiplicative structures of the set of integers. The reader is referred to [Ba4] for a more thorough discussion of this problem than what is possible in these pages. We merely comment here that this problem seems to be difficult and wide open (see [Sh] for best available result), although even asymptotic results are available in certain circumstances (see [El] and [Hi]). Furthermore, our new results in this direction, although sufficient for the purposes of proving Theorems 1 and 2 and take up the bulk of the paper [Ba3], fall far short of (11) and only imply that, under certain hypotheses on the range of parameters involved, (11) holds true on average. Nevertheless, it seems quite plausible that such an estimate should hold under rather mild assumptions on $g$ and in a wide range of $q$. In particular, we believe that, analogously to Conjecture 1, the condition $S(x) \gg \log _{2} x$ should go a long way towards guaranteeing that (11) holds. We thus pose the following conjecture (apparently first formulated in [Ba4]).

Conjecture 2. Let $\epsilon, 0<\epsilon \leq 1 / 2$, be fixed. Then estimate (11) with the implied constant depending only on $\epsilon$ holds uniformly for all $(a, q)=1, q \leq x^{1-\epsilon}$, and all $g \in \mathcal{G}_{\epsilon}(x)$, where, analogously to $\mathcal{F}_{\lambda}(x), \mathcal{G}_{\lambda}(x)$ is the subclass of $\mathcal{G}$ consisting of functions $g$ satisfying $S(x) \geq \lambda \log _{2} x$.

As we have mentioned already, Corollary 2 provides a slight improvement on (4) even in the case when $S(x)$ is maximal or, more generally, when $e^{S(x)} \asymp \log x$. For this important special case we can offer further improvements as follows.

Theorem 3. Let $x \geq 3$ and $\alpha$ be real numbers and set $Q=x /(\log x)^{3}$. Furthermore, let $a$ and $q$ be coprime integers satisfying $q \leq Q$ and $|\alpha-a / q| \leq 1 /(q Q)$. Then we have

$$
F(x, \alpha) \ll \frac{x}{\log x}+\frac{x}{\sqrt{\varphi}(q)},
$$

uniformly for all $f \in \mathcal{F}$.

Theorem 4. Let $x \geq 3, \alpha$ and $R \geq 3$ be real numbers and suppose that $|\alpha-s / r| \leq$ $1 / r^{2}$ and $R \leq r \leq x / R$ for some coprime integers $s$ and $r$. Then we have

$$
F(x, \alpha) \ll \frac{x}{\log x}+\frac{x}{\sqrt{R}}\left(\log R \log _{2} R\right)^{1 / 2},
$$

uniformly for all $f \in \mathcal{F}$.

\section{REFERENCES}

[Ba1] G. Bachman, On the coefficients of cyclotomic polynomials, Mem. Amer. Math. Soc. 510 (1993). MR 94d:11068

[Ba2] - On exponential sums with multiplicative coefficients, Number theory (Halifax, NS, 1994), CMS Conf. Proc., 15, Amer. Math. Soc., Providence, RI, 1995, pp. 29-38. MR 96i:11091

[Ba3] - On exponential sums with multiplicative coefficients, II, in preparation. 
[Ba4] Some remarks on non-negative multiplicative functions on arithmetic progressions, J. Number Theory 73 (1998), 72-91. MR 99k:11142

[Da1] H. Daboussi, Fonctions multiplicatives presque périodiques B. D'après un travail commun avec Hubert Delange, Journées Arithmétique de Bordeaux (Conf. Univ. Bordeaux, 1974), Astérisque 24-25 (1975), 321-324. MR 51:10274

[Da2] On some exponential sums, Analytic number theory (Allerton Park, IL, 1989), Progr. Math., 85 (B. C. Berndt, H. G. Diamond, H. Halberstam and A. Hildebrand, eds.), Birkhäuser, Boston, MA, 1990, pp. 111-118. MR 92a:11092

[DD1] H. Daboussi and H. Delange, Quelques propriétés des fonctions multiplicatives de module au plus égal à 1, C.R. Acad. Sci. Paris Ser. A 278 (1974), 657-660. MR 48:11028

[DD2] - On multiplicative arithmetic functions whose modulus does not exceed one, J. London Math. Soc. (2) 26 (1982), 245-264. MR 83k:10082

[Da] H. Davenport, On some infinite series involving arithmetic functions II, Quart. J. Math. Oxford 8 (1937), 313-320.

[DHT] Y. Dupain, R. R. Hall et G. Tenenbaum, Sur l'équirépartition modulo 1 de certaines fonctions de diviseurs, J. London Math. Soc. (2) 26 (1982), 397-411. MR 84m:10047

[El] P. D. T. A. Elliott, Multiplicative functions on arithmetic progressions. VI. More middle moduli, J. Number Theory 44 (1993), 178-208. MR 94i:11073

[FT] E. Fouvry et G. Tenenbaum, Entiers sans grand facteur premier en progressions arithmétiques, Proc. London. Math. Soc. 63 (1991), 449-494. MR 93c:11074

[HR] H. Halberstam and H.-E. Richert, On a result of R. R. Hall, J. Number Theory 11 (1979), 76-89. MR 80j: 10050

[Ha] R. R. Hall, Halving an estimate obtained from Selberg's upper bound method, Acta Arith. 25 (1974), 347-351. MR 49:4949

[Hi] A. Hildebrand, Multiplicative functions on arithmetic progressions, Proc. Amer. Math. Soc. 108 (1990), 307-318. MR 90d:11112

[HT] A. Hildebrand and G. Tenenbaum, Integers without large prime factors, J. Théorie des Nombres de Bordeaux 5 (1993), 411-484. MR 95d:11116

[Go] L. Goubin, Sommes d'exponentielles et principe de l'hyperbole, Acta Arith. 73 (1995), 303-324. MR 96i:11089

[MV1] H. L. Montgomery and R. C. Vaughan, The order of magnitude of the mth coefficients of cyclotomic polynomials, Glasgow Math. J. 27 (1985), 143-159. MR 87e:11026

[MV2] _ Exponential sums with multiplicative coefficients, Invent. Math. 43 (1977), 69-82. MR 56:15579

[Sh] P. Shiu, A Brun-Titchmarsh Theorem for multiplicative functions, J. reine angew. Math. 313 (1980), 161-170. MR 81h:10065

[Te] G. Tenenbaum, Facteurs premiers de sommes d'entiers, Proc. Amer. Math. Soc. 106 (1989), 287-296. MR 89k:11091

[Va1] R.C. Vaughan, A new iterative method in Waring's problem, Acta Math. 162 (1989), 1-71. MR 90c: 11072

[Va2] , Private communication.

Department of Mathematical Sciences, University of Nevada, Las Vegas, 4505 MaryLAND PARKWAy, Las Vegas, Nevada 89154-4020

E-mail address: bachman@nevada.edu 